Portland State University

PDXScholar

$11-1-1972$

\title{
Decay of Multiple Spin Echoes in Dipolar Solids
}

C. H. Wang

The University Of Utah

John D. Ramshaw

Portland State University, jdramshaw@yahoo.com

Follow this and additional works at: https://pdxscholar.library.pdx.edu/phy_fac

Part of the Physics Commons

Let us know how access to this document benefits you.

\section{Citation Details}

C.H. Wang and J.D. Ramshaw, "Decay of Multiple Spin Echoes in Dipolar Solids," Phys. Rev. B 6, 3253 (1972)

This Article is brought to you for free and open access. It has been accepted for inclusion in Physics Faculty Publications and Presentations by an authorized administrator of PDXScholar. Please contact us if we can make this document more accessible: pdxscholar@pdx.edu. 
lattices increases a few degrees below $T_{c}$. Critical superparamagnetism cannot therefore be the cause of the $\beta=\frac{1}{2}$ exponent found near $T_{c}$ in magnetization measurements.

${ }^{53}$ J. M. D. Coey, G. A. Sawatzky, and A. H. Morrish, Phys. Rev. 184, 334 (1969).

${ }^{54}$ G. K. Wertheim, H. J. Guggenheim, and D. N. E. Buchanan, Phys. Rev。 169, 465 (1968).

${ }^{55}$ J. M. D. Coey, D. C. Price, and A. H. Morrish, Rev. Sci. Instr. 43, 54 (1972).

${ }^{56}$ L。 Néel, Ann. Phys. $\underline{3}, 317$ (1948).

${ }^{57}$ R. Aleonard, J. C. Barbier, and R. Pauthenet, Comp. Rend. Acad. Sci. Paris 242, 2531 (1956).
${ }^{58} \mathrm{~K}$. Miyatani and K. Yoshikawa, J. Appl. Phys. 41 , $1272(1970)$.

${ }^{59} \mathrm{Y}$. Ishikawa, J. Appl. Phys. 35, 1054 (1964).

${ }^{60} \mathrm{~J}$. M. D. Coey and D. Khalafalla, Phys. Status Solidi a11, 229 (1972); J. M. D. Coey, Phys. Rev. Letters 27,1140 (1971).

${ }^{61}$ Weak fields may decrease the relaxation time.

[A. Aharoni and R. Bijaoui (unpublished).]

${ }^{62}$ S. Geller, Phys. Rev. 181, 980 (1969),

${ }^{63}$ I. Nowik, Phys. Rev. 171, 550 (1968); J. Appl. Phys. 40, 5184 (1969).

${ }^{64} \mathrm{D}$. Lebenbaum and I. Nowik, Phys. Letters 31A, 373 (1970).

\title{
Decay of Multiple Spin Echoes in Dipolar Solids
}

\author{
C. H. Wang \\ Department of Chemistry, The University of Utah, Salt Lake City, Utah 84112 \\ and \\ John D. Ramshaw \\ Department of Physics, The University of Utah, Salt Lake City, Utah 84112 \\ (Received 27 December 1971)
}

\begin{abstract}
In this paper we derive a general expression describing the evolution of the transverse nuclear-spin magnetization for the Ostroff-Waugh multiple-spin-echo experiment in dipolar solids. Our approach consists of expressing the formula for the magnetization at even echoes in a form resembling an ordinary time-correlation function, and then evaluating this quantity by means of Zwanzig's projection-operator technique. For long times, we show that under certain conditions the echo envelope decays exponentially, in agreement with experiment. A general expression is obtained for the time constant $T^{*}$ associated with the decay. This result may be used to generate an expansion of $1 / T^{*}$ in powers of the cycle time $t_{c}$, but there are experimental indications that this expansion is not legitimate and that more complicated $t_{c}$ dependences can arise. In the case when higher-order correlations decay much more rapidly than lower-order ones, our result reduces to $1 / T^{*}=A t_{c}^{4} \tau_{c}^{0}\left(t_{c}\right)$, where $A$ is a quantity related to the sixth moment of the magnetization and $\tau_{c}^{0}\left(t_{c}\right)$ is a characteristic correlation time associated with decay of the lowest-order correlation function which enters the problem. The $t_{c}$ dependence of $T^{*}$ is then determined by the behavior of $\tau_{c}^{0}\left(t_{c}\right)$, and is in general more complex than the proportionality between $1 / T^{*}$ and $t_{c}^{5}$ found previously. This previous result emerges in the case when $\tau_{c}^{0}\left(t_{c}\right)=t_{c}$. Available experimental results suggest that $1 / T^{*}$ is in general a nonanalytic function of $t_{c}$, as indicated by the observed proportionality between $1 / T^{*}$ and $t_{c}$ for Teflon and $\mathrm{KAsF}_{6}$. Further experimental results are needed to clarify the nature of this nonanalytic behavior.
\end{abstract}

\section{INTRODUCTION}

It was reported ${ }^{1,2}$ that a periodic train of $90^{\circ} \mathrm{rf}$ pulses can greatly prolong the decay of transverse nuclear spin magnetization in dipolar solids. The effect is observed as a train of multiple spin echoes analogous to (but quite different in character from) the familiar "classical" spin echoes first observed by Hahn. ${ }^{3}$ A detailed analysis of this effect, including the dependence of the decay time $T^{*}$ for the echo envelope upon the pulse spacing, was given by Waugh and Wang. ${ }^{4}$ The pulse sequence which gives rise to the effect may be represented symbolically as $P_{x}, \tau, P_{y},\left(2 \tau, P_{y}\right)_{n}$, where
$P_{\alpha}(\alpha=x, y)$ denotes a $90^{\circ}$ pulse along the $\alpha$ axis of a reference frame rotating at the Larmor frequency $\omega_{0}\left(=\gamma H_{0}\right)$. The first pulse $P_{x}$ serves merely to establish a suitable initial condition for the remainder of the sequence. This pulse is followed, after a time $\tau$, by a train of $n P_{y}$ pulses $\left(n \sim 10^{3}\right)$ spaced apart by a time $2 \tau$.

The free induction decay following a single $P_{x}$ pulse in a dipolar solid decays nonexponentially to zero on a time scale $T_{2}\left(T_{2}\right.$ is the normal transverse relaxation time). However, the echo train induced by the action of the subsequent $P_{y}$ pulses persists for times several orders of magnitude longer than $T_{2},{ }^{1,2,4}$ In fact, by reducing the pulse 
spacing the time constant $T^{*}$ characterizing the decay of the echo envelope can be made to approach $T_{1 \rho}$, the spin-lattice relaxation time in the rotating frame. ${ }^{1,4}$

As mentioned above, the multiple spin echoes under consideration here are of a completely different character from the ordinary spin echoes used to recover decay caused by static inhomogeneous interactions. These ordinary spin echoes are normally observed in liquids, and depend for their occurrence upon a distribution in Larmor frequencies due to such causes as magnetic field inhomogeneities, quadrupole coupling, dipolar coupling to nonresonant spins, and so on. In contrast, the multiple spin echoes induced in dipolar solids by a train of $90^{\circ}$ pulses are more complex in nature, being governed by the manner in which the $N$-body magnetic-dipole-dipole interactions (and other terms in the spin Hamiltonian) transform under the action of the pulses.

By exploiting the symmetry which the dipolar Hamiltonian exhibits under the influence of two successive $90^{\circ}$ pulses, Waugh and Wang ${ }^{4}$ were able to show that for short times (i.e., $n \tau \approx T_{2}$ ) the decay of the echo envelope is proportional to $n^{2}$. However, at long times (i.e., $n \tau \gg T_{2}$ ) the echo envelope is observed to decay exponentially in a variety of samples. ${ }^{4-7}$ It is therefore clear that the longer-time behavior cannot properly be accounted for by a naive projection of the short-time behavior, for such a projection would imply a Gaussian rather than an exponential decay. Recognizing this difficulty, Waugh and Wang attempted to calculate the long-time behavior of the echo envelope by combining the calculated decay of the magnetization after one cycle $(n=2)$ with an Ansatz (similar in spirit to the familiar Stosszahlansatz of Boltzmann) concerning the manner in which this decay accumulates at long times. According to this argument, the time constant $T^{*}$ which characterizes the exponential decay of the echo envelope should be proportional to $\tau^{-5}$. It is of interest to note that in at least two samples $\left(\mathrm{CaF}_{2}\right.$ and $\left.\mathrm{K}_{2} \mathrm{SiF}_{6}\right)$ $T^{*}$ is observed to have precisely this $\tau$ dependence. ${ }^{4}$ The agreement between theory and experiment in the case of $\mathrm{CaF}_{2}$ was subsequently confirmed even more strongly by Mansfield and Ware. ${ }^{6}$

However, the proportionality of $T^{*}$ to $\tau^{-5}$ for this experiment is by no means universal. Experimental data for Teflon and $\mathrm{KAsF}_{6}$ indicate a proportionality between $T^{*}$ and $\tau^{-1}{ }^{5}$ In other samples still different dependences of $T^{*}$ upon $\tau$ are observed. We mention in particular the recent work of Mansfield, Richards, and Ware ${ }^{7}$ (MRW) on solid samples containing more than one spin species. MRW report that their experimental data cannot be explained on the basis of the simple $a d$ hoc argument used by Waugh and Wang. In order to account for their experimental results, MRW present a modification of Anderson's theory of spectral-line narrowing in solids. ${ }^{8}$ Although they are able to fit their observations into the framework of this theory, their work does not appear to clarify substantially the manner in which different dependences of $T^{*}$ upon $\tau$ might manifest themselves in different circumstances.

The purpose of the present paper is to derive a general expression for the decay time $T^{*}$. In principle, this expression contains the full dependence of $T^{*}$ upon $\tau$. In this paper we shall restrict attention to the case in which only a single spin species is present. The generalization to several spin species will be considered in a subsequent publication. Our method of approach is to cast the problem into a form which resembles an ordinary time-correlation-function calculation, and then to evaluate the resulting expression using the projection-operator technique developed by Zwanzig. ${ }^{9}$ Our results indicate that $T^{*}$ may exhibit a variety of different $\tau$ dependences depending upon the circumstances. One possibility is that $1 / T^{*}$ may be expanded in powers of $\tau$. The coefficients in this expansion may easily be generated from our basic result for $T^{*}$. Unfortunately, however, there are experimental indications that such an expansion is frequently not legitimate. The reason for this is unclear; it may be that experimentally accessible values of $\tau$ are not "small enough" in the required sense, or it may be due to some inherent nonanalyticity in the problem. In any event, if the expansion of $1 / T^{*}$ in powers of $\tau$ is not legitimate, then the manner in which $T^{*}$ depends upon $\tau$ is critically dependent upon the properties of the correlation function which appears in the expression for $T^{*}$ [see Eq. (34)]. This correlation function is of course too complicated to permit a direct evaluation; the best that can be done is to consider several possible forms for it and examine the $\tau$ dependence to which each gives rise. We do this in connection with our discussions of experimental data, and indicate how the original $\tau^{-5}$ dependence, as well as other $\tau$ dependences, may arise.

Although our main concern in this paper is with the $P_{x}, \tau, P_{y},\left(2 \tau, P_{y}\right)_{n}$ pulse sequence, our method may equally well be applied to analyze the decay times for other pulse sequences. It will be remembered that the principal significance of the prolonged decay time in the Ostroff-Waugh experiment ${ }^{1}$ was the line narrowing which it implied in frequency space. Motivated by the possibility this suggested for accomplishing "high-resolution" NMR in solids, Waugh and co-workers ${ }^{10-15}$ soon discovered that the decay of the magnetization in a wide class of multiple-pulse experiments could 
be simply understood by introducing the concept of an "average Hamiltonian" which is determined by the symmetry properties of the true Hamiltonian under the pulse transformations. Once this concept became clearly understood, it became possible to design pulse sequences specifically to suppress the dipole-dipole interactions, enabling the structure associated with more interesting interactions (such as chemical shifts) to be resolved. A number of experiments of this type were quickly proposed and successfully performed..$^{10-17}$

Although the line-narrowing capabilities of such experiments can be analyzed in terms of the average Hamiltonian and its higher-order correction terms, ${ }^{13}$ we feel that the approach of this paper may have some complementary usefulness in this regard. In fact, our work may be regarded as a natural extension of that of Waugh and co-workers. They have considered in great detail the form of the average Hamiltonian and its low-order correction terms for a variety of multiple-pulse experiments. Our approach goes one step further and considers explicitly the corresponding time decay of the echo envelope. In order that our work may be applicable to a variety of multiple-pulse experiments, we present the basic theory in a form which is independent of the particular pulse sequence under consideration (so long as it is cyclic). We then specialize our results to the case of particular interest in this paper.

\section{THEORY}

1. Evolution of the transverse spin magnetization under the action of a train of $r f$ pulses. We consider the evolution of a system under the influence of a time-dependent Hamiltonian $\mathfrak{F}(t)$. This evolution is most compactly described in terms of the density operator $\rho(t)$, which satisfies the von Neumann-Liouville equation

$$
i \hbar \frac{\partial \rho}{\partial t}=[\mathfrak{H C}(t), \rho(t)] \text {. }
$$

In the rotating-coordinate frame, the time dependence of $\mathfrak{F C}(t)$ is due to a cyclic train of $\mathrm{rf}$ pulses applied in the plane normal to the static magnetic field. (Nonsecular terms oscillating at multiples of the Larmor frequency also arise when the transformation to the rotating frame is made, but their effects are unimportant for our purposes so these terms are neglected.) Also contained in $\mathcal{H}(t)$ is a time-independent internal Hamiltonian $\mathcal{F}_{\text {int }}$. In this paper we shall neglect spin-lattice relaxation and consider the lattice to be rigid. In this case, $\mathfrak{F}_{\mathrm{int}_{\mathrm{t}}}$ is a time-independent function of spin variables. It also depends, of course, on lattice variables (such as the distance $r_{i j}$ between spins $i$ and $j$ ), but these are present only as constant parameters. Typical contributions to $\mathcal{F}_{\mathfrak{i n t}}$ for solids in- clude dipole-dipole interactions, scalar couplings, chemical shifts, and so on. In conventional NMR experiments in solids, the dipole-dipole interactions give rise to a broad, featureless spectrum, making it impossible to extract information concerning the other interactions. Multiple-pulse experiments are of interest because they make possible the suppression of the dipole-dipole interactions, the reby allowing the resolution of spectral details associated with the other interactions.

In general, the solution to Eq. (1) involves a time-ordering operator, ${ }^{13}$ which is necessary to make proper allowance for the interference between the internal Hamiltonian and the specific rf pulse sequence used in the experiment. We shall consider the situation in which the rf pulse field, when present, is much greater than the internal dipolar field. This situation is readily achieved in multiple-pulse experiments. In this case the effect of a pulse is essentially that of a $\delta$ function; the pulse may then be represented by a rotation operator in the rotating frame. Our specific concern in this paper is with $90^{\circ}$ pulses; the corresponding rotation operator simply effects a $90^{\circ}$ rotation of the spin operators along a predetermined direction in the rotating frame.

Experimentally, of course, the pulses are not really $\delta$ functions, and in some cases the effects of finite pulse width become important. Haeberlen and Waugh ${ }^{13}$ have showed how to allow for these effects.

Before the application of the rf pulses, the spin system is at equilibrium and is described by the density operator $\rho_{e q}=Z^{-1} \exp \left[-\beta\left(\mathcal{H}_{z}+\mathcal{H}_{i n t}\right)\right]$, where $\mathcal{F}_{Z}$ is the Zeeman Hamiltonian and $Z$ is the partition function. In the high-temperature approximation, $\rho_{\text {eq }}$ can be written

$$
\begin{aligned}
\rho_{\mathrm{eq}} & \approx\left(\operatorname{Tr} 1_{\mathrm{op}}\right)^{-1}\left[1_{\mathrm{op}}-\beta\left(\mathcal{H} \mathcal{C}_{z}+\mathcal{H} \mathcal{H}_{\mathrm{int}}\right)\right] \\
& \approx\left(\operatorname{Tr} 1_{\mathrm{op}}\right)^{-1}\left[1_{\mathrm{op}}+\beta \hbar \omega_{0} I_{z}\right],
\end{aligned}
$$

where $\omega_{0}=\gamma H_{0}$ is the Larmor frequency. The internal Hamiltonian may be neglected in the hightemperature approximation because its contribution is thousands of times smaller than the Zeeman term in the experiments considered here. Now $\operatorname{Tr} 1_{o p}=(2 I+1)^{N}$ is simply a constant, and the unit operator on the right-hand side of Eq. (2) makes no contribution to the magnetization (since angular momentum operators are traceless). Therefore, the useful or significant part of the equilibrium density operator before the rf pulsing is simply proportional to $I_{z}$.

In all multiple-pulse experiments, the first $90^{\circ}$ pulse $P_{x}$ serves to prepare a state with nonzero transverse magnetization. After this initial $90^{\circ}$ pulse the density operator therefore becomes proportional to $I_{y}$. For the purpose of calculating the 
transverse magnetization after the ensuing coherent set of pulses, it is convenient to consider $I_{y}$ as an initial condition: $\rho(0)=I_{y}$. The subsequent evolution of $\rho(0)$ is then governed by the internal Hamiltonian and the remainder of the rf pulses. Thus, the density operator which describes the free induction decay at a time $\tau$ after the first pulse $P_{x}$ is

$$
\rho(\tau)=\mathscr{D}(\tau) I_{y} D^{-1}(\tau),
$$

where

$$
D(\tau)=\exp \left(-i \mathcal{F}_{\mathrm{int}} \tau\right) .
$$

In Eq. (4) we have adopted the convention of writing $\mathcal{F C}_{\text {Int }}$ in units of angular frequency, which is equivalent to setting $\hbar=1$. This convention will be observed throughout the remainder of this paper.

But instead of observing the free induction decay, we apply another pulse $P_{1}$ at a time $\tau=\tau_{0}$. We then let the system evolve freely for a time $\tau_{1}$, and then apply another pulse $P_{2}$. We continue in this manner until a sequence of $N$ pulses $\left\{P_{1} P_{2} \cdots P_{N}\right\}$ has been applied, the spacing between successive pulses $P_{i}$ and $P_{i+1}$ being denoted by $\tau_{i}$. At a time $\tau_{N}$ after the pulse $P_{N}$ the density operator has become

$$
\rho_{N}=K I_{y} K^{-1},
$$

where

$$
K=\mathscr{D}\left(\tau_{N}\right) P_{N} D\left(\tau_{N-1}\right) P_{N-1} \cdots P_{1} D\left(\tau_{0}\right) .
$$

There exists no simple way of calculating $\rho_{N}$ for an arbitrary rf pulse sequence with random pulse spacings. However, a considerable simplification results if (a) the pulse sequence is designed in such a way that a set of $m$ successive pulses $(m \ll N)$ recovers the original Hamiltonian, i.e.,

$$
P_{j+m} \cdots P_{j+1} \mathcal{H}_{\text {int }} P_{j+1}^{-1} \cdots P_{j+m}^{-1}=\mathcal{H C}_{\mathrm{int}} ;
$$

(b) the pulse spacings are so adjusted that the pulse sequence becomes periodic in time, each period $t_{c}$ containing $m$ pulses. Pulse sequences satisfying these conditions are said to be cyclic $^{10,13}$; the period $t_{c}$ is called the cycle time, and is equal to $\tau_{0}+\tau_{1}+\cdots+\tau_{m}$.

If the pulse sequence is cyclic and if $N$ is an integral multiple of $m$ (say $N=n m$ ), then Eq. (5) reduces to

$$
\rho_{N}(N=n m)=L^{n}\left(t_{c}\right) I_{y} L^{-n}\left(t_{c}\right),
$$

where the one-cycle propagator $L\left(t_{c}\right)$ is given by

$$
L\left(t_{c}\right)=\mathfrak{D}\left(\tau_{m}\right) P_{m} \mathfrak{D}\left(\tau_{m-1}\right) \cdots P_{1} \mathfrak{D}\left(\tau_{0}\right) .
$$

The $y$ component (in the rotating frame) of the transverse magnetization at the end of the $n$th pulse cycle is then proportional to

$$
\left\langle I_{y}\left(n t_{c}\right)\right\rangle=\operatorname{Tr}\left[I_{y} L^{n}\left(t_{c}\right) I_{y} L^{-n}\left(t_{c}\right)\right] .
$$

The one-cycle propagator $L\left(t_{c}\right)$ defined in Eq. (9) can be rewritten in the form ${ }^{10}$

$$
\begin{aligned}
& L\left(t_{c}\right)=\left[D\left(\tau_{m}\right) D_{m}\left(\tau_{m-1}\right) \mathscr{D}_{m, m-1}\left(\tau_{m-2}\right) \ldots\right. \\
& \left.\times D_{m, m-1}, \cdots, 1\left(\tau_{0}\right)\right]\left(P_{m} P_{m-1} \cdots P_{2} P_{1}\right),
\end{aligned}
$$

where

$$
D_{i, j}, \ldots(\tau)=\exp \left[-i \tau\left(P_{i} P_{j} \cdots \mathcal{H}_{\mathrm{int}} \cdots P_{j}^{-1} P_{i}^{-1}\right)\right] .
$$

Things become simpler if we now restrict attention to cycles such that

$$
P_{m} P_{m-1} \cdots P_{2} P_{1} f(I)=e^{i \phi} f(I),
$$

where $\phi$ is some real number and $f(I)$ is any function of spin variables. Equation (13) is clearly sufficient to guarantee that Eq. (7) is satisfied; it is also necessary if Eq. (7) is to be satisfied for arbitrary $\mathfrak{H}_{\mathrm{int}}$, since any operator which commutes with every other operator must be proportional to the unit operator. Now if Eq. (13) is satisfied, then the pulse operators $\left(P_{m} P_{m-1} \cdots P_{2} P_{1}\right)$ in Eq. (11) cancel out when this equation is substituted into either Eq. (8) or (10). For the purpose of calculating $\left\langle I_{y}\left(n t_{c}\right)\right\rangle$, therefore, Eq. (11) may be replaced by

$$
L\left(t_{c}\right)=D\left(\tau_{m}\right) D_{m}\left(\tau_{m-1}\right) \cdots D_{m, m-1, \ldots,}\left(\tau_{0}\right) .
$$

It is convenient to define a $t_{c}$-dependent effective Hamiltonian $\mathcal{F}_{e}\left(t_{c}\right)$ by the equation

$$
L\left(t_{c}\right)=\exp \left[-i t_{c} \mathfrak{F C}_{e}\left(t_{c}\right)\right] .
$$

Since $L\left(t_{c}\right)$ is unitary, $\mathcal{F}_{e}\left(t_{c}\right)$ must be Hermitian. It may be evaluated in series form by means of the Magnus expansion, ${ }^{13,18,19}$ which yields

$$
\mathfrak{H C}_{e}\left(t_{c}\right)=\overline{\mathfrak{H}}+\sum_{k=1}^{\infty} \overline{\mathfrak{H}}^{(k)}\left(t_{c}\right),
$$

together with a prescription for determining the quantities $\overline{\mathfrak{H}}$ and $\overline{\mathfrak{H}}^{(k)}\left(t_{c}\right)$. The quantity $\overline{\mathcal{H}}$ is called the average Hamiltonian; as indicated by our notation it is independent of $t_{c}$. If the pulse spacings are fixed fractions of $t_{c}$ then $\overline{\mathcal{F}}^{(k)}\left(t_{c}\right)=t_{c}^{k} F_{k}$, where $F_{k}$ is a Hermitian operator independent of $t_{c}$. The expansion in Eq. (16) is therefore simply a power series in $t_{c}$. The quantities $F_{k}$ can be determined either by the Magnus prescription or simply by expanding both Eqs. (14) and (15) in powers of $t_{c}$ and equating coefficients.

We see from Eqs. (15) and (16) that in the limit $t_{c} / T_{2} \rightarrow 0, n \rightarrow \infty$, and $n t_{c}=$ const $L^{n}\left(t_{c}\right)$ approaches $\exp \left(-i n t_{0} \overline{H C}\right)$ exactly. Historically, ${ }^{10}$ this fact was realized before it became clear how to introduce correction terms which allow for finite $t_{c}$. These correction terms are important, however, since the mathematical limit $t_{c} / T_{2}$ is of course not realized in practice. In the present context, these corrections are of vital importance to the calculation of the decay of $\left\langle I_{y}\left(n t_{c}\right)\right\rangle$, since if they were 
omitted there would be no decay of the magnetization. ${ }^{10}$

The above development is essentially a capsule summary of what has become known as average Hamiltonian theory. We have included it both in order to make our presentation self-contained and to avoid notational inconsistencies. Since it is probably too condensed to serve as an introduction to the subject, the reader desiring further details is referred to the original sources..$^{4,10-15}$

2. Exact integral equation for the transverse magnetization. According to Eq. (15), the $n$-cycle propagator which develops the system for a time $n t_{c}$ is simply equal to $\exp \left[-i n t_{c} \mathcal{H}_{e}\left(t_{c}\right)\right]$. Equation (10) therefore becomes

$\left\langle I_{y}\left(n t_{c}\right)\right\rangle=\operatorname{Tr}\left\{I_{y} \exp \left[-i n t_{c} \mathfrak{F}_{e}\left(t_{c}\right)\right] I_{y} \exp \left[i n t_{c} \mathfrak{H C}_{e}\left(t_{c}\right)\right]\right\}$.

Equation (17) now appears in a form resembling an ordinary time-correlation function. Having put $\left\langle I_{y}\left(n t_{c}\right)\right\rangle$ into this form, we are now in a position to evaluate it using the projection-operator technique introduced into statistical mechanics by Zwanzig. ${ }^{9}$ At first there appears to be a problem, because Eq. (17) is valid only for integral values of $n$; that is, only for times which are an integral multiple of the cycle time $t_{c}$. In order to avoid the problems associated with a discrete variable, we simply define a continuous function $G(t)$ of the continuous variable $t$ by

$$
\begin{aligned}
G(t) & \equiv \operatorname{Tr}\left\{I_{y} \exp \left[-i t \mathcal{C}_{e}\left(t_{c}\right)\right] I_{y} \exp \left[i t \mathcal{H}_{e}\left(t_{c}\right)\right]\right\} \\
& \equiv \operatorname{Tr}\left\{I_{y} I_{y}(t)\right\} \equiv\left\langle I_{y} I_{y}(t)\right\rangle .
\end{aligned}
$$

We then proceed to calculate the evolution of $G(t)$ as $t$ is varied continuously. At the end of the calculation, we can regain $\left\langle I_{y}\left(n t_{c}\right)\right\rangle$ by setting $t=n t_{c}$ in $G(t)$, i.e., $\left\langle I_{y}\left(n t_{c}\right)\right\rangle=G\left(n t_{c}\right)$. In this manner we can obtain rigorous information about the spin magnetization at integral multiples of the cycle time $t_{c}$, even though $G(t)$ does not correspond to the magnetization at other times.

It is, of course, understood that $G(t)$ depends parametrically upon $t_{c}$ as well as upon $t$, even though the former dependence has been suppresse for notational convenience. Since we shall calculate $G(t)$ by varying only $t, t_{c}$ remains constant throughout our development and we need not worry about it.

We first differentiate $I_{y}(t)$ with respect to $t$, obtaining

$$
\frac{\partial I_{y}(t)}{\partial t}=i\left[\mathfrak{H}_{e}\left(t_{c}\right), I_{y}(t)\right] \equiv i \mathscr{L}\left(t_{c}\right) I_{y}(t),
$$

with the initial condition $I_{y}(0)=I_{y}$. Equation (19) is formally identical to the Heisenberg equation of motion for the operator $I_{y}$ in a system who se timeindependent Hamiltonian is equal to $\mathfrak{H}_{e}\left(t_{c}\right)$. We have introduced, in Eq. (19), the Liouville opera- tor $\mathcal{L}\left(t_{c}\right)$ corresponding to the effective Hamiltonian $\mathcal{H}_{e}\left(t_{c}\right) . \mathcal{L}\left(t_{c}\right)$ is a superoperator, which yields the commutator $\left[\mathcal{H C}_{e}\left(t_{c}\right), A\right]$ when it operates on any operator $A$.

We are not really interested in the complete solution to Eq. (19); we want only the component of $I_{y}(t)$ which is necessary to calculate $G(t)$. Following Zwanzig, ${ }^{9}$ we call this component the relevant part of $I_{y}(t)$ and denote it by $I_{1}(t)$. This component can be separated out by the use of a projection operator $\odot$ defined as follows:

$$
\odot A=\left\langle I_{y} A\right\rangle\left\langle I_{y}^{2}\right\rangle^{-1} I_{y},
$$

where $A$ is any quantum-mechanical operator. It is easy to show from this definition that $\beta$ is idempotent, i.e., $\rho^{2}=\odot$. We therefore write

$$
I_{1}(t)=\odot I_{y}(t)=\left\langle I_{y} I_{y}(t)\right\rangle\left\langle I_{y}^{2}\right\rangle^{-1} I_{y} \text {. }
$$

The other part of $I_{y}(t)$ (i. e., the irrelevant component) will be called $I_{2}(t)$, so that

$$
I_{y}(t)=\odot I_{y}(t)+(1-\odot) I_{y}(t)=I_{1}(t)+I_{2}(t) \text {. }
$$

As shown by Zwanzig, ${ }^{9}$ the above equations imply in a straightforward manner that $I_{1}(t)$ satisfies the following integro-differential equation:

$$
\begin{aligned}
\frac{\partial I_{1}(t)}{\partial t}=\rho i \mathcal{L}\left(t_{c}\right)\left\{I_{1}(t)+\int_{0}^{t}\right. & d t^{\prime} \exp \left[\left(t-t^{\prime}\right)(1-\rho) i \mathcal{L}\left(t_{c}\right)\right] \\
& \left.\times(1-\rho) i \mathcal{L}\left(t_{c}\right) I_{1}\left(t^{\prime}\right)\right\}
\end{aligned}
$$

where the fact that $(1-\rho) I_{y}(0)=0$ has been used to eliminate a term which would otherwise appear.

Now notice that

$$
\begin{aligned}
\rho \mathcal{L}\left(t_{c}\right) I_{1}(t) & =\rho \mathcal{L}\left(t_{c}\right) I_{y}\left\langle I_{y}^{2}\right\rangle^{-1} G(t) \\
& =\operatorname{Tr}\left\{I_{y}\left[\mathcal{H}_{e}\left(t_{c}\right), I_{y}\right]\right\}\left\langle I_{y}^{2}\right\rangle^{-2} G(t) I_{y},
\end{aligned}
$$

which vanishes because the trace is invariant to cyclic permutation. Thus the first term on the right-hand side of Eq. (23) is zero. If we now multiply both sides of Eq. (23) by $I_{y}$ and take the trace, we obtain

$$
\begin{aligned}
\frac{\partial G(t)}{\partial t} & =-\int_{0}^{t} d t^{\prime}\left\langle I_{y}^{2}\right\rangle^{-1}\left\langle I_{y} \mathcal{L}\left(t_{c}\right)\right. \\
& \left.\times \exp \left[\left(t-t^{\prime}\right)(1-\mathcal{\beta}) i \mathcal{L}\left(t_{c}\right)\right] \mathcal{L}\left(t_{c}\right) I_{y}\right\rangle G\left(t^{\prime}\right),
\end{aligned}
$$

which is an exact integro-differential equation for $G(t)$. If this equation can be solved, formally or otherwise, then an exact equation for $\left\langle I_{y}\left(n t_{c}\right)\right\rangle$ can be obtained immediately by setting $t=n t_{c}$. Thus, Eq. (24) completely determines the evolution of the transverse magnetization at the end of the $n \mathrm{th}$ pulse cycle.

\section{APPLICATION TO OSTROFF-WAUGH EXPERIMENT}

In this section we shall apply the preceding results to analyze the Ostroff-Waugh experiment ${ }^{1}$ for 
production of multiple dipolar echoes in solids containing only a single spin species. ${ }^{2,6}$ Further applications to the case of several spin species and to other pulse sequences designed to accomplish high-resolution NMR in solids will be discussed in a subsequent publication. For the case considered here, we will rederive the short-time result of Waugh and Wang from Eq. (24), and will show as well that under certain conditions this equation simplifies to imply an exponential decay of the magnetization at long times. When this is the case, an explicit but formal expression for the decay time $T^{*}$ is automatically obtained. The resulting dependence of $T^{*}$ upon $t_{c}$ (or $\tau$ ) can be quite varied depending upon the circumstances; we dis cuss briefly the manner in which different $\tau$ dependences may arise.

1. Specialization to the $P_{x}, \tau, P_{y},\left(2 \tau, P_{y}\right)_{n}$ pulse cycle. The one-cycle propagator corresponding to the Ostroff-Waugh pulse sequence is ${ }^{4}$

$$
L\left(t_{c}\right)=\exp \left(-i \mathcal{H}_{d} \tau\right) \exp \left(-2 i \tilde{\mathcal{F}}_{d} \tau\right) \exp \left(-i \mathcal{H}_{d} \tau\right),
$$

where $t_{c}=4 \tau, \tilde{H}_{d}=P_{y} \mathcal{H}_{d} P_{y}^{-1}$, and $\mathcal{H}_{d}$ is the truncated dipolar Hamiltonian appropriate to solids:

$$
\mathcal{H}_{d}=\sum_{i<j} \gamma^{2} \hbar r_{i j}^{-3} P_{2}\left(\cos \theta_{i j}\right)\left(\vec{I}_{i} \cdot \vec{I}_{j}-3 I_{i z} I_{j z}\right) \text {. }
$$

For simplicity, we consider the case in which the internal Hamiltonian contains only dipole-dipole interactions, but scalar couplings can be trivially included if desired; nothing essential is changed.

Since the one-cycle propagator given in Eq. (25) is symmetrical [i.e., $L^{\dagger}\left(t_{c}\right)=L\left(-t_{c}\right)$ ], all the oddorder correction terms in Eq. (16) vanish (see Appendix). Therefore,

$L\left(t_{c}\right)$
where

$$
L\left(t_{c}\right)=\exp \left[-i t_{c} \mathfrak{H}_{e}\left(t_{c}\right)\right],
$$

$$
\mathfrak{H}_{e}\left(t_{c}\right)=\overline{\mathcal{H}}+\sum_{k=1}^{\infty} t_{c}^{2 k} F_{2 k} \equiv \overline{\mathcal{H}}+V\left(t_{c}\right) .
$$

The first two nonvanishing terms in Eq. (28) involve the quantities $\overline{\dddot{K}}$ and $F_{2}$, which are given by

$$
\begin{aligned}
& \overline{\mathcal{H}}=\frac{1}{4} i L_{1}=\frac{1}{2}\left(\mathcal{H C}_{d}+\tilde{\mathcal{H}}_{d}\right), \\
& F_{2}=\left(i / 4^{3}\right)\left[L_{3}-(1 / 3 !) L_{1}^{3}\right],
\end{aligned}
$$

where $L_{k}$ is the coefficient of $\tau^{k}$ in a power-series expansion of $L(4 \tau)$ [Eq. (27)].

So far we have not questioned the convergence of the Magnus expansion in Eqs. (16) and (28). The expansion is certainly expected to converge rapidly if $t_{c}$ is small enough, but in practice this may not always be the case. In any event, we shall consider $V\left(t_{c}\right)$ to be defined by $\mathfrak{H}_{e}\left(t_{c}\right)-\overline{\mathfrak{H}}$, whether or not the expansion in Eq. (28) is convergent.

We next note that $\left[\overline{\mathfrak{H}}, I_{y}\right]=0$, which in turn immediately implies that $\rho[\overline{\mathcal{F}}, A]=0$ for any operator $A$. Making use of these facts and Eq. (28), we finally obtain

$$
\frac{\partial G(t)}{\partial t}=-\int_{0}^{t} d t^{\prime} K\left(t^{\prime} \mid t_{c}\right) G\left(t-t^{\prime}\right),
$$

where

$$
\begin{aligned}
K\left(t^{\prime} \mid t_{c}\right) & =\left\langle I_{y}^{2}\right\rangle^{-1}\left\langle I_{y} \mathcal{L}^{\prime}\left(t_{c}\right)\right. \\
& \left.\times \exp \left[i \mathscr{L}_{0} t^{\prime}+i(1-\odot) \mathcal{L}^{\prime}\left(t_{c}\right) t^{\prime}\right] \mathcal{L}^{\prime}\left(t_{c}\right) I_{y}\right\rangle,
\end{aligned}
$$

$\mathcal{L}_{0} A \equiv[\overline{\mathcal{H}}, A]$, and $\mathscr{L}^{\prime}\left(t_{c}\right) A \equiv\left[V\left(t_{c}\right), A\right]$ for any operator $A$. Equations (29) and (30) completely determine the evolution of the magnetization, and are valid for both short and long times. These equations are closely analogous to Terwiel and Mazur's expressions for the spin-spin relaxation function. ${ }^{20}$ The quantities $\overline{\mathcal{H}}$ and $V\left(t_{c}\right)$ of the present case play, respectively, the roles of secular and nonsecular Hamiltonians. Of course, the nonsecular perturbation in the present case is introduced by the fact that the characteristic repetition period $t_{c}$ is finite. If $t_{c} \ll T_{2}$, the nonsecular effects become negligibly small and the motion of the system can be described in terms of the average Hamiltonian $\overline{\mathfrak{H}}$. In this case the magnetization will be locked along the $y$ direction of the rotating frame ${ }^{4}$; i. e., there will be no decay of the echo envelope. This situation is of course similar to the familiar "motional narrowing" effect, as has been discussed by Haeberlen and Waugh. ${ }^{13}$

2. Behavior at short times. We wish here to show that the above results reduce for short times to the results of Waugh and Wang. ${ }^{4}$ For small $\tau$ we have, from Eq. (28),

$$
\begin{aligned}
V\left(t_{c}\right) & =t_{c}^{2} F_{2}+O\left(t_{c}^{4}\right) \\
& =\frac{1}{4} i \tau^{2}\left[L_{3}-(1 / 3 !) L_{1}^{3}\right]+O\left(\tau^{4}\right),
\end{aligned}
$$

and we obtain for short times and small $\tau$, from Eq. (30),

$$
\begin{aligned}
& K\left(t^{\prime} \mid t_{c}\right)=t_{c}^{4}\left\langle I_{y}\left[F_{2},\left[F_{2}, I_{y}\right]\right]\right\rangle\left\langle I_{y}^{2}\right\rangle^{-1}+O\left(t_{c}^{6}\right) \\
& K\left(t^{\prime} \mid \tau\right)=\left(i^{2} / 4^{2}\right) \tau^{4}\left\langle I_{y}\left[L_{3},\left[L_{3}, I_{y}\right]\right]\right\rangle\left\langle I_{y}^{2}\right\rangle^{-1} .
\end{aligned}
$$$$
\text { or }
$$

In terms of the brace notation of Waugh and Wang, ${ }^{4}$ this equation becomes

$$
K\left(t^{\prime} \mid \tau\right)=\left(i^{2} / 4^{2}\right) \tau^{4} \llbracket L_{3}^{2} \rrbracket / \llbracket 1 \rrbracket .
$$

Substituting Eq. (32) into Eq. (29), solving for $G(t)$ to lowest order in $t$, and setting $t=n t_{c}=4 n \tau$, we obtain

$$
\left\langle I_{y}\left(n t_{c}\right)\right\rangle=\left\langle I_{y}(4 n \tau)\right\rangle=\llbracket 1+\frac{1}{2} n^{2} \tau^{6} L_{3}^{2}+\cdots \rrbracket,
$$

which is identical to Eq. (41) of Waugh and Wang. ${ }^{21}$

3. Behavior at long times. We now wish to examine the implications of our basic equations (29) and (30) for long times, i. e., for $n t_{c} \gg T_{2}$. To do so, we observe that while the decay function $G(t)$ decays slowly to zero on a time scale $T_{1 \rho}$, the kernel 
$K\left(t^{\prime} \mid t_{c}\right)$ [which consists essentially of the time autocor relation function of the quantity $\mathcal{L}^{\prime}\left(t_{c}\right) I_{y}$, with a modified propagator] is expected to decay rapidly to zero in a time of the order of $T_{2}{ }^{22}$ If this is the case, then for $T_{2} \ll n t_{c} \ll T_{1 \rho}$, one may approximate Eq. (29) by setting the upper integra- tion limit equal to $\infty$ and replacing $G\left(t-t^{\prime}\right)$ by $G(t)$. When this approximation is made, the function $G(t)$ (and hence the magnetization) assumes an exponential decay, which in fact is what is observed experimentally at long times. The time constant $T^{*}$ associated with the decay is then given by

$$
\frac{1}{T^{*}}=-\int_{0}^{\infty} d t^{\prime} \frac{\left\langle\left[\mathcal{L}^{\prime}\left(t_{c}\right) I_{y}\right] \exp \left[i \mathscr{L}_{0} t^{\prime}+i(1-\mathcal{Q}) \mathcal{L}^{\prime}\left(t_{c}\right) t^{\prime}\right]\left[\mathcal{L}^{\prime}\left(t_{c}\right) I_{y}\right]\right\rangle}{\left\langle I_{y}^{2}\right\rangle}
$$

where, in spite of the minus sign, $1 / T^{*}$ must of course be positive. It is perhaps more revealing to write $1 / T^{*}$ as

$$
\frac{1}{T^{*}}=-\frac{\left\langle\left[\mathcal{L}^{\prime}\left(t_{c}\right) I_{\nu}\right]^{2}\right\rangle}{\left\langle I_{\nu}^{2}\right\rangle} \tau_{c}\left(t_{c}\right)
$$

where the characteristic correlation time $\tau_{c}\left(t_{c}\right)$ associated with the decay of the kernel is given by

$$
\tau_{c}\left(t_{c}\right)=\int_{0}^{\infty} d t^{\prime} \frac{\left\langle\left[\mathcal{L}^{\prime}\left(t_{c}\right) I_{y}\right] \exp \left[i \mathscr{L}_{0} t^{\prime}+i(1-\Phi) \mathcal{L}^{\prime}\left(t_{c}\right) t^{\prime}\right]\left[\mathcal{L}^{\prime}\left(t_{c}\right) I_{y}\right]\right\rangle}{\left\langle\left[\mathcal{L}^{\prime}\left(t_{c}\right) I_{y}\right]^{2}\right\rangle}
$$

Equation (34) [or Eqs. (35) and (36)] must contain the full dependence of $T^{*}$ upon $t_{c}$, although the precise nature of this dependence has yet to be extracted from the formalism. Let us therefore go on to consider particular circumstances and the $t_{c}$ dependence which arises in each.

In the limit of small $t_{c}\left(t_{c} \ll T_{2}\right)$, it is reasonable to suppose that Eq. (34) reduces to

$$
1 / T^{*}=-t_{c}^{4}\left\langle I_{y}^{2}\right\rangle^{-1} \int_{0}^{\infty} d t^{\prime}\left\langle\left[F_{2}, I_{y}\right] \exp \left(i \mathscr{L}_{0} t^{\prime}\right)\left[F_{2}, I_{y}\right]\right\rangle .
$$

We emphasize, however, that in order to obtain Eq. (37) from Eq. (34) in the limit $t_{c} / T_{2} \rightarrow 0$ it is necessary to interchange limiting operations in a manner which has not been mathematically justified. ${ }^{23}$ Equation (37) bears an obvious resemblance to the well-known expressions for the ordinary spin-lattice and spin-spin relaxation times $T_{1}$ and $T_{2}{ }^{24}$ Note that according to Eq. (37) $1 / T^{*}$ should be proportional to $t_{c}^{4}$ (or $\tau^{4}$ ) for small $t_{c}$. This result is in disagreement with experimental results for $\mathrm{CaF}_{2}$, which show a proportionality between $T^{*}$ and $t_{c}^{-5}$, although it may account for the departure from this relationship in the case of $\mathrm{K}_{2} \mathrm{SiF}_{6}$ at small $t_{c}$.

In practice, however, the mathematical limit $t_{c} / T_{2} \rightarrow 0$ is never achieved, and we must consider the question of how to include correction terms in Eq. (37) arising from the fact that $t_{c}$ is finite. The simplest way to do this is to assume that it is legitimate to express $1 / T^{*}$ as a power series in $t_{c}$. This expansion can be generated in a straightfor ward manner from the basic expression (34) for $1 / T^{*}$. One first expands the exponential operator in powers of $\mathcal{L}^{\prime}\left(t_{c}\right)$, allowing, of course, for the fact that the operators in the exponent do not nec- essarily commute. One then expands $\mathcal{L}^{\prime}\left(t_{c}\right)$, wherever it appears, in powers of $t_{c}$; since $\mathscr{L}^{\prime}\left(t_{c}\right) A$ $=\left[V\left(t_{c}\right), A\right]$, this expansion is essentially the same as that in Eq. (28). But since only even powers of $t_{c}$ appear in Eq. (28), it is clear that only even powers can appear in $1 / T^{*}$ as well. That is, if $1 / T^{*}$ may be expanded in powers of $t_{c}$, then the expansion begins with a term of order $t_{c}^{4}$, and only even powers of $t_{c}$ appear:

$$
1 / T^{*}=a_{1} t_{c}^{4}+a_{2} t_{c}^{6}+a_{3} t_{c}^{8}+\cdots,
$$

where $a_{1}$ is of course just the negative of the integral appearing in Eq. (37).

But there is a serious difficulty with this powerseries approach, for experimentally it is found in several cases that $1 / T^{*}$ is proportional to $t_{c}^{5}$. According to Eq. (38), 1/T* should start out propor tional to $t_{c}^{4}$, and even if the $t_{c}^{4}$ term should happen to be zero, the next term would be proportional to $t_{c}^{6}$ rather than $t_{c}^{5}$. One possible explanation for this disagreement is the following. Suppose it turns out that, although the $t_{c}^{4}$ and $t_{c}^{6}$ terms in Eq. (38) are important, all higher-order terms are negligible in the range of $t_{c}$ considered. Then Eq. (38) may be rewritten in the form

$$
1 / T^{*}=t_{c}^{5}\left(a_{1} / t_{c}+a_{2} t_{c}\right) \text {. }
$$

If we suppose further that $a_{1} \approx a_{2} t_{c}^{2}$ in the range of $t_{c}$ considered, then we see from Eq. (39) that it is possible for the combination of a $t_{c}^{4}$ term and a $t_{c}^{6}$ term to simulate the behavior of $t_{c}^{5}$ over a reasonably wide range of $t_{c}$. Of course, this argument is purely conjectural; it rests upon the above suppositions concerning the relative magnitudes of the coefficients $a_{k}$ in Eq. (38). In order to justify this interpretation, it would be necessary to evaluate 


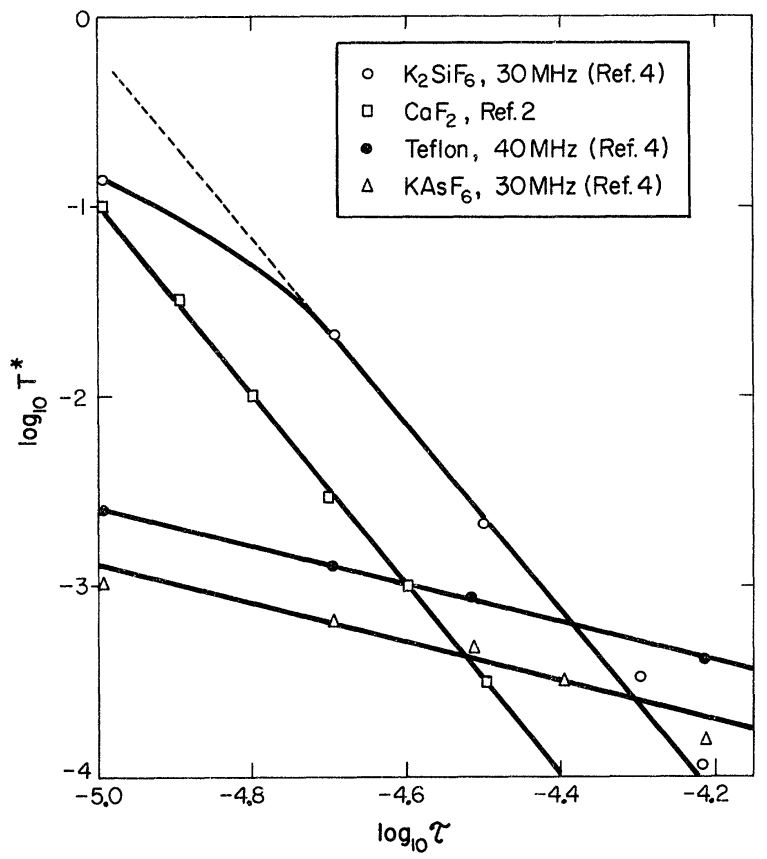

FIG. 1. Dependence of the decay time $T^{*}$ on pulse spacing $\tau\left(\tau=t_{c} / 4 ; t_{c}\right.$ is the cycle time $)$ in $\mathrm{K}_{2} \mathrm{SiF}_{6}, \mathrm{CaF}_{2}$, $\mathrm{KAsF}_{6}$, and Teflon. These data were obtained from Figs. 2 and 3 of Ref. 4. Note that $T^{*} \propto \tau^{-5}$ for $\mathrm{CaF}_{2}$ and $\mathrm{K}_{2} \mathrm{SiF}_{6}$, while $T^{*} \propto \tau^{-1}$ for $\mathrm{KAsF}_{6}$ and Teflon.

the quantities $a_{k}$; this is unfortunately a prohibitive task. Besides, even if this explanation is correct for $\mathrm{CaF}_{2}$ and $\mathrm{K}_{2} \mathrm{SiF}_{6}$ its applicability is in no sense general, for there are several other compounds which show other dependences of $T^{*}$ upon $t_{c}$ (see Fig. 1). In particular, for Teflon and $\mathrm{KAsF}_{6}$ one finds a proportionality between $1 / T^{*}$ and $t_{c}$; there is no apparent way to reconcile these observations with Eq. (38).

The above difficulties suggest that it simply may not be permissible to express $1 / T^{*}$ as a power series in $t_{c}$, at least in the experimentally accessible range of $t_{c}$. This may occur because experimentally $t_{c}$ is not made small enough for the series to converge rapidly or at all, or it may be due to some inherent nonanalyticity in the problem. That is to say, $1 / T^{*}$ may simply not be an analytic function of $t_{c}$ over an interval containing $t_{c}=0$. But if the power-series expansion of $1 / T^{*}$ is not legitimate, then it becomes apparent that $1 / T^{*}$ may exhibit practically any $t_{c}$ dependence that one can imagine. In particular, the proportionality between $1 / T^{*}$ and $t_{c}^{5}$ which is observed for $\mathrm{CaF}_{2}$ and $\mathrm{K}_{2} \mathrm{Si}_{6}$ may arise in the following manner. Let us first suppose that $t_{c}$ is small enough for the Magnus expansion (28) to converge, which is almost certainly the case. However, the fact that experimentally $t_{c} \lesssim T_{2}$ (instead of the more favorable condition $t_{c}$ $\left.\ll T_{2}\right)$ means that we cannot expect to be able to truncate the expansion at any low order. If we now expand $\mathcal{L}^{\prime}\left(t_{c}\right)$ in powers of $t_{c}$ everywhere except in the exponential, Eq. (34) becomes

$$
\begin{aligned}
\frac{1}{T^{*}} & =-t_{c}^{4} \int_{0}^{\infty} d t^{\prime} \frac{\left\langle M_{2} E\left(t^{\prime} \mid t_{c}\right) M_{2}\right\rangle}{\left\langle I_{y}^{2}\right\rangle} \\
- & t_{c}^{6} \int_{0}^{\infty} d t^{\prime} \frac{\left\langle M_{4} E\left(t^{\prime} \mid t_{c}\right) M_{2}+M_{2} E\left(t^{\prime} \mid t_{c}\right) M_{4}\right\rangle}{\left\langle I_{y}^{2}\right\rangle}+\ldots,
\end{aligned}
$$

where $M_{k} \equiv\left[F_{k}, I_{y}\right]$ and $E\left(t^{\prime} \mid t_{c}\right) \equiv \exp \left[i \mathscr{L}_{0} t^{\prime}+i(1-\beta)\right.$ $\left.\times \mathcal{L}^{\prime}\left(t_{c}\right) t^{\prime}\right]$. In obtaining Eq. (40) from Eq. (34) we have effectively assumed that the difficulty in expressing $1 / T^{*}$ as a power series in $t_{c}$ is associated with the exponential operator rather than the rest of the expression.

As mentioned, we cannot expect to be able to truncate Eq. (40) at any low order on the basis of the smallness of $t_{c}$. However, it is reasonable physically to assume that the progressively higherorder correlation functions which occur in succeeding terms of Eq. (40) decay to zero with increasing rapidity. ${ }^{8}$ If this is the case, then we can truncate the expansion in Eq. (40) on this basis regardless of the fact that $t_{c} \lesssim T_{2}$. We then obtain

$$
1 / T^{*}=-\left(\left\langle M_{2}^{2}\right\rangle /\left\langle I_{y}^{2}\right\rangle\right) t_{c}^{4} \tau_{c}^{0}\left(t_{c}\right),
$$

where $\tau_{c}^{0}\left(t_{c}\right)$ is given by

$$
\tau_{c}^{0}\left(t_{c}\right)=\int_{0}^{\infty} d t^{\prime} \frac{\left\langle M_{2} E\left(t^{\prime} \mid t_{c}\right) M_{2}\right\rangle}{\left\langle M_{2}^{2}\right\rangle}
$$

and is presumed not to be expressible as a power series in $t_{c}$. We see from Eq. (41) that if $1 / T^{*}$ is to be proportional to $t_{c}^{5}$ then $\tau_{c}^{0}\left(t_{c}\right)$ must simply be proportional to $t_{c}$. This will occur if the integrand of Eq. (42) [which is the time autocorrelation function of the operator $M_{2}$, taken with the modified propagator $E\left(t^{\prime} \mid t_{c}\right)$ and normalized to unity at $t^{\prime}=0$ ] is flat up to a time $t^{\prime}=\alpha t_{c}$ (where $\alpha$ is a constant), beyond which it assumes a very rapid decay. (Actually, all that is necessary is that the area be equal to $\alpha t_{c}$, but this is perhaps less easily imagined.) Then $\tau_{c}^{0}\left(t_{c}\right) \approx \alpha t_{c}$ and one obtains

$$
1 / T^{*}=-\alpha\left(\left\langle M_{2}^{2}\right\rangle /\left\langle I_{y}^{2}\right\rangle\right) t_{c}^{5} .
$$

If $\alpha$ is set equal to unity, we obtain the earlier result of Waugh and Wang, ${ }^{4}$ obtained by use of a Stosszahlansatz.

The proportionality between $1 / T^{*}$ and $t_{c}$ which is observed for Teflon and $\mathrm{KAsF}_{6}$ may be interpreted in the same manner. In order for Eq. (41) to imply that $1 / T^{*}$ is proportional to $t_{c}$, it is necessary for $\tau_{c}^{0}\left(t_{c}\right)$ to be proportional to $t_{c}^{-3}$. The corresponding behavior of the correlation function in the integrand of Eq. (42) may readily be imagined. ${ }^{25}$ If, for example, this correlation function decays exponentially with a time constant proportional to $t_{c}^{-3}$ then $\tau_{c}^{0}\left(t_{c}\right)$ is, of course, proportional to $t_{c}^{-3}$. 
This is a sufficient condition but certainly not a necessary one.

\section{CONCLUSION}

We have seen that a theory of the decay of transverse spin magnetization in multiple-pulse NMR experiments can be formulated in close analogy to theories of more conventional relaxation experiments. In particular, our treatment makes clear the close connection which exists between the limit $\left(t_{c} \rightarrow 0, n \rightarrow \infty, n t_{c}=t=\right.$ const $)$ in multiple-pulse NMR and the familiar weak-coupling limit of statisical mechanics. ${ }^{9}$

We then focused attention on the Ostroff-Waugh experiment for producing multiple spin echoes in dipolar solids, and obtained a formal expression for the decay time $T^{*}$ which in principle contains the full dependence of this quantity upon the pulse spacing $\tau$. Guided by available experimental data, we gave a discussion of the different possible dependences of $T^{*}$ upon $\tau$ which may arise in different circumstances. In order to obtain agreement with experiment, the correlation function which determines $T^{*}$ [see Eq. (34)] must apparently manifest different types of unusual behavior in different samples. However, experimental data of this type are very scarce (to our knowledge, Fig. 1 is a complete summary of such data for samples containing a single spin species). Further measurements of the dependence of $T^{*}$ upon $\tau$, both for the Ostroff-Waugh experiment and for other pulse sequences, would be very helpful in the resolution of the questions we have raised. We hope that this work will serve as a stimulus for further experi- mental investigations of the dependence of $T^{*}$ upon the pulse spacing.

\section{ACKNOWLEDGMENT}

We thank Professor John S. Waugh for his careful reading of the manuscript and for his comments on the paper.

\section{APPENDIX} (16):

For convenience we rewrite here Eqs. (15) and

$$
\begin{aligned}
& L\left(t_{c}\right)=\exp \left[-i t_{c} \mathcal{H}_{e}\left(t_{c}\right)\right], \\
& \mathcal{H}_{e}\left(t_{c}\right)=\overline{\mathcal{H}}+\sum_{k=1}^{\infty} t_{c}^{k} F_{k},
\end{aligned}
$$

where the fact that $\overline{\mathcal{F}}^{(k)}\left(t_{c}\right)=t_{c}^{k} F_{k}$ has been used. Since $L\left(t_{c}\right)$ is a unitary operator, it follows that

$$
L^{\dagger}\left(t_{c}\right)=L^{-1}\left(t_{c}\right)=\exp \left[i t_{c} \mathfrak{H}_{e}\left(t_{c}\right)\right] .
$$

If $L\left(t_{c}\right)$ is symmetrical as well, that is, if

$$
L^{\dagger}\left(t_{c}\right)=L\left(-t_{c}\right)
$$

then one also has

$$
\exp \left[i t_{c} \mathcal{H}_{e}\left(t_{c}\right)\right]=\exp \left[i t_{c} \mathcal{H}_{e}\left(-t_{c}\right)\right] \text {. }
$$

Therefore,

$$
\mathfrak{H}_{e}\left(t_{c}\right)=\mathfrak{H}_{e}\left(-t_{c}\right)
$$

or, from Eq. (A2),

$$
\sum_{k=1}^{\infty} t_{c}^{k} F_{k}=\sum_{k=1}^{\infty}\left(-t_{c}\right)^{k} F_{k}
$$

Therefore, $F_{k}$ must be equal to zero if $k$ is odd.
${ }^{1} \mathrm{E}$. D. Ostroff and J. S. Waugh, Phys. Rev. Letters 16, 1097 (1966).

${ }^{2}$ P. Mansfield and D. Ware, Phys. Letters 22, 133 (1966).

${ }^{3}$ E. L. Hahn, Phys. Rev. 80,580 (1950).

${ }^{4}$ J. S. Waugh and C. H. Wang, Phys. Rev. 162, 209 (1967).

${ }^{5} \mathrm{~J}$. S. Waugh (private communication).

${ }^{6}$ P. Mansfield and D. Ware, Phys. Rev. 168, 318 (1968).

${ }^{7}$ P. Mansfield, K. H. B. Richards, and D. Ware, Phys. Rev. B 1,2048 (1970).

${ }^{8}$ P. W. Anderson, J. Phys. Soc. Japan 9 , 314 (1954).

${ }^{9} \mathrm{R}$. W. Zwanzig, in Lectures in Theoretical Physics, edited by W. E. Brittin, B. W. Downs, and J. Downs (Interscience, New York, 1961), Vol. III, p. 106.

${ }^{10}$ J. S. Waugh, C. H. Wang, L. M. Huber, and R. L. Vold, J. Chem. Phys. 48, 662 (1968).

${ }^{11}$ J. S. Waugh and L. M. Huber, J. Chem. Phys. 47, 1862 (1967).

${ }^{12}$ J. S. Waugh, L. M. Huber, and U. Haeberlen, Phys. Rev. Letters 20, 180 (1968).

${ }^{13} \mathrm{U}$. Haeberlen and J. S. Waugh, Phys. Rev. $\underline{175}, 453$ (1968).

${ }^{14}$ U. Haeberlen and J. S. Waugh, Phys. Rev. $\underline{185}$,
420 (1969).

${ }^{15}$ J. D. Ellett, Jr., U. Haeberlen, and J. S. Waugh, Polymer Letters $\underline{7}, 71$ (1969).

${ }^{16} \mathrm{U}$. Haeberlen, J. D. Ellett, Jr., and J. S. Waugh, J. Chem. Phys. 55, 53 (1971).

${ }^{17}$ L. M. Stacey, R. W. Vaughan, and D. D. Elleman, Phys. Rev. Letters 26, 1153 (1971).

${ }^{18}$ W. Magnus, Commun. Pure Appl. Math. 7, 649 (1954).

${ }^{19}$ (a) P. Pechukas and J. C. Light, J. Chem. Phys. 44, 3897 (1966); (b) W. A. B. Evans, Ann. Phys.

(N. Y.) 48,72 (1968).

${ }^{20} \mathrm{R}$. H. Terwiel and P. Mazur, Physica $\underline{32}, 1813$ (1966).

${ }^{21}$ Equation (42) of Ref. 4 should read $2 \llbracket L_{6} \rrbracket=\llbracket L_{3}^{2} \rrbracket$, instead of $\llbracket L_{6} \rrbracket=2 \llbracket L_{3}^{2} \rrbracket$.

${ }^{22}$ A rigorous demonstration that $K\left(t^{\prime} \mid t_{c}\right)$ decays rapidly would be prohibitively difficult because of the highorder correlations involved. However, this rapid decay is plausible on the basis of the similarity in structure between $K\left(t^{\prime} \mid t_{c}\right)$ and the correlation function associated with $T_{2}$ processes in solids. In addition, if one expands $K\left(t^{\prime} \mid t_{c}\right)$ in powers of $t^{\prime}$ then it is found that the lowestorder contribution to the coefficient of $\left(t^{\prime}\right)^{k}$ is a modified $k$ th moment of a type which is frequently taken in the 
literature [see Refs. 4, 13, and 19(b)] to be of the order of $T_{2}^{-k}$. This indicates that the relevant time parameter is $t^{\prime} / T_{2}, \mathrm{i}$.e., that the decay of $K\left(t^{\prime} \mid t_{c}\right)$ takes place on a time scale $T_{2}$. But regardless of these qualitative arguments, the rapid decay of $K\left(t^{\prime} \mid t_{c}\right)$ is necessary in order that the decay of $G(t)$ be exponential, as observed experimentally.

${ }^{23}$ Both the infinite sum which defines the exponential operator and the infinite integral are associated with limiting processes, so the passage from Eq. (34) to Eq. (37) in the limit as $t_{c} / T_{2} \rightarrow 0$ is by no means as obvious as it may at first appear. See Ref. 20 for a related discussion.

${ }^{24}$ J. M. Deutch and I. Oppenheim, in Advances in Magnetic Resonance, edited by J. S. Waugh (Academic, New York, 1968), Vol. 3, p. 43.

${ }^{25}$ One might wonder whether the inclusion of lattice motion in the theory would be capable of explaining a proportionality between $1 / T^{*}$ and $t_{c}$. We have made some preliminary calculations of this type, and find that the inclusion of lattice motion does not appear to alter the situation materially. In particular, the leading term in a power-series expansion of $1 / T^{*}$ is still proportional to $t_{c}^{4}$, just as in the present case [see Eq. (38)].

\title{
Proton Nuclear Magnetic Relaxation in Antiferromagnetic $\mathrm{NiCl}_{2} \cdot 6 \mathrm{H}_{2} \mathrm{O}, \mathrm{CoCl}_{2} \cdot 6 \mathrm{H}_{2} \mathrm{O}$, $\mathrm{CuCl}_{2} \cdot 2 \mathrm{H}_{2} \mathrm{O}$, and $\mathrm{MnBr}_{2} \cdot 4 \mathrm{H}_{2} \mathrm{O}^{+*}$
}

\author{
I. J. Lowe and D. W. Whitson $\$ \$$ \\ Department of Physics, University of Pittsburgh, Pittsburgh, Pennsylvania 15213 \\ (Received 17 May 1972)
}

\begin{abstract}
Proton-spin-lattice relaxation times $\left(T_{1}\right)$ were measured by us in antiferromagnetic $\mathrm{NiCl}_{2} \cdot 6 \mathrm{H}_{2} \mathrm{O}\left(T_{N}=5.34^{\circ} \mathrm{K}\right), \mathrm{CoCl}_{2} \cdot 6 \mathrm{H}_{2} \mathrm{O}\left(T_{N}=2.29^{\circ} \mathrm{K}\right)$, and $\mathrm{MnBr}_{2} \cdot 4 \mathrm{H}_{2} \mathrm{O}\left(T_{N}=2.13^{\circ} \mathrm{K}\right)$ in the temperature range $4.18-1.12^{\circ} \mathrm{K}$. Spin-echo experiments were also carried out over the same temperature range to determine the homogeneous transverse relaxation time $T_{2}$ in $\mathrm{NiCl}_{2} \cdot 6 \mathrm{H}_{2} \mathrm{O}$ and the extent of the inhomogeneous broadening in $\mathrm{CoCl}_{2} \cdot 6 \mathrm{H}_{2} \mathrm{O}$. Samples of various impurities and shapes were investigated. The spin-lattice relaxation time $T_{1}$ was found to be strongly temperature dependent for the first two crystals. The $T_{1}$ data from the $\mathrm{CoCl}_{2} \cdot 6 \mathrm{H}_{2} \mathrm{O}$ crystals could be fit to a power law $\left(T_{1} \propto T^{-7}\right)$, but the $T_{1}$ data from the $\mathrm{NiCl}_{2} \cdot 6 \mathrm{H}_{2} \mathrm{O}$ crystals could not. The temperature dependence of $T_{1}$ for $\mathrm{MnBr}_{2} \cdot 4 \mathrm{H}_{2} \mathrm{O}$ was not determined because of the short temperature range over which we were able to make measurements. The temperature dependence of $T_{1}$ for the first two crystals along with the experimental results for $\mathrm{CuCl}_{2} \cdot 2 \mathrm{H}_{2} \mathrm{O}\left(T_{N}\right.$ $=4.3^{\circ} \mathrm{K}$ ) have been explained through a first-order two-magnon process plus a second-order three-magnon process. The second-order three-magnon process arises from the four-magnon exchange interaction which has been shown by Beeman and Pincus to completely supersede the first-order three-magnon process when $T>T_{A E}$. The impurity levels, the sample shape, and the orientation of an external magnetic field $H_{0}$ had no effect on the temperature dependence of $T_{1}$. In $\mathrm{NiCl}_{2} \cdot 6 \mathrm{H}_{2} \mathrm{O}$ the value of $T_{2}$ measured from a $90^{\circ}-90^{\circ}$-pulse-sequence experiment increased from a value of about $28 \mu \mathrm{sec}$ at $4.18^{\circ} \mathrm{K}$ to a value of about $65 \mu \mathrm{sec}$ at $2.3^{\circ} \mathrm{K}$ and then at $2.1^{\circ} \mathrm{K}$ dropped to about $30 \mu \mathrm{sec}$ and stayed at this value down to $1.12^{\circ} \mathrm{K}$. In $\mathrm{CoCl}_{2}{ }^{\circ} 6 \mathrm{H}_{2} \mathrm{O}$ the inhomogeneous broadening was dependent on the orientation of $H_{0}$ and the sample shape. An rf enhancement was found in $\mathrm{CoCl}_{2} \cdot 6 \mathrm{H}_{2} \mathrm{O}$ for $\mathrm{H}_{0}$ along the anisotropy axis ( $c$ axis).
\end{abstract}

\section{INTRODUCTION}

The first experimental measurements of the spin-lattice relaxation time $T_{1}$ for protons in antiferromagnetic materials were made by Hardeman et $a l .{ }^{1}$ Moriya $^{2}$ and Van Kranendonk and Bloom ${ }^{3}$ tried to explain the dependence of $T_{1}$ on the absolute temperature $T$ using a relaxation process due to the Raman scattering of magnons. Their results were of the right order of magnitude, but the temperature dependence was slower than the $T^{-7}$ dependence found experimentally. Pincus and Winte $\mathrm{r}^{4}$ then proposed a theory which gave the $T^{-7}$ temperature dependence but required $T \ll T_{A E}$, where $k_{B} T_{A E}=\hbar \omega_{A E}$ and $\hbar \omega_{A E}$ is the magnon energy gap. They assumed that the magnetostrictive terms in the Hamiltonian produced a magnon component in the thermal phonon spectrum allowing the phonons to participate directly in the relaxation processes. The $T^{-7}$ temperature dependence in $\mathrm{CuCl}_{2} \cdot 2 \mathrm{H}_{2} \mathrm{O}\left(T_{A E} \simeq 1.4^{\circ} \mathrm{K}\right)$ covers the range $T$ $>T_{A E}$ as well as the range $T<T_{A E}$. Other crystals, $\mathrm{CoCl}_{2} \cdot 6 \mathrm{H}_{2} \mathrm{O}\left(T_{A E} \simeq 2{ }^{\circ} \mathrm{K}\right)$ and $\mathrm{KMnF}_{3}\left(T_{A E} \simeq 0.3^{\circ} \mathrm{K}\right)$, that have also exhibited this temperature dependence for $T_{1}$ have also included the range $T>T_{A E}$. In fact for $\mathrm{KMnF}_{3}$ the middle of the temperature range is on the order of 100 times $T_{A E}$. Also, as is reported in Sec. IV, the temperature dependence of $T_{1}$ in $\mathrm{NiCl}_{2} \cdot 6 \mathrm{H}_{2} \mathrm{O}$ cannot be fitted by a power law. Additional arguments and evidence against 